\title{
Assessment of Efficacy of Proarrhythmia Biomarkers in Isolated Rabbit Hearts With Attenuated Repolarization Reserve
}

\author{
Szabolcs Orosz, MSc, * Annamária Sarusi, MD, † Norbert Csík, PhD, ł Julius Gy. Papp, MD, PhD, DSc, †\$ \\ András Varró, MD, PhD, DSc, †§ Sándor Farkas, MD, * Tamás Forster, MD, PhD, DSc, $\emptyset$ \\ Attila S. Farkas, MD, PhD, ๆ and András Farkas, MD, PhD
}

\begin{abstract}
Isolated hearts with reduced repolarization reserve would be suitable for assessing the proarrhythmic liability of drugs. However, it is not known which proarrhythmia biomarkers indicate the increased susceptibility to torsades de pointes arrhythmia (TdP) in such experimental setting. Thus, we estimated the efficacy of proarrhythmia biomarkers in isolated hearts with attenuated repolarization reserve. Langendorff-perfused rabbit hearts were used. Repolarization reserve was reduced by concomitant inhibition of the rapid $\left(\mathrm{I}_{\mathrm{Kr}}\right)$ and slow $\left(\mathrm{I}_{\mathrm{Ks}}\right)$ delayed rectifier potassium currents by dofetilide and HMR-1556, respectively. Rate corrected QT (QTc) interval and beat-to-beat variability of the QT interval measured in sinus rhythm or irrespective of rhythm even during arrhythmias (sinus and absolute QT variability, respectively) were tested. QTc failed to predict increased proarrhythmic risk. Sinus QT variability indicated proarrhythmic liability when low concentration of dofetilide was used. However, when arrhythmias compromised sinus variability measurement during coperfusion of catecholamines and elevated concentration of dofetilide, only absolute QT variability indicated increased proarrhythmic risk. Absolute QT variability parameters seem to be the most practical and sensitive biomarkers of proarrhythmic liability in rabbit hearts with reduced repolarization reserve. Absolute QT variability parameters could serve as surrogates for torsades de pointes in drug-safety investigations in isolated rabbit hearts with attenuated repolarization reserve.
\end{abstract}

Received for publication January 21, 2014; accepted April 18, 2014.

From the ${ }^{*}$ Gedeon Richter Plc., Budapest, Hungary; $†$ Department of Pharmacology and Pharmacotherapy, University of Szeged, Szeged, Hungary; †Department of Electrical Engineering and Cybernetics, Kecskemét College, Kecskemét, Hungary; §Division of Cardiovascular Pharmacology, Hungarian Academy of Sciences, Szeged, Hungary; and $\uparrow$ Second Department of Medicine and Cardiology Centre, Faculty of Medicine, University of Szeged, Szeged, Hungary.

A. S. Farkas and A. Farkas equally contributed and shared senior authorship. The authors report no conflicts of interest.

Supported by Hungarian Scientific Research Fund (OTKA PD 105882, OTKA NK-104331), National Development Agency and European Regional Fund (TÁMOP-4.2.2-08/1-2008-0013, TÁMOP-4.2.1/B-09/1/KONV-2010-0005, TÁMOP-4.2.2/B-10/1-2010-0012, TÁMOP-4.2.2.A-11/1/KONV-2012-0052, TÁMOP-4.2.2.A-11/1/KONV-2012-0060, TÁMOP-4.2.2.A-11/1/KONV2012-0073), Hungary-Romania Cross-Border Cooperation Program (HURO/1001/086/2.2.1_HURO-TWIN). A. S. Farkas is a holder of János Bolyai fellowship (BO/00794/12) of the Hungarian Academy of Sciences.

Reprints: Attila S. Farkas, MD, PhD, Second Department of Medicine and Cardiology Centre, Faculty of Medicine, University of Szeged, Korányi fasor 6, H-6720 Szeged, Hungary (e-mail: farkasamed@gmail.com).

Copyright (C) 2014 by Lippincott Williams \& Wilkins
Key Words: absolute beat-to-beat variability parameters, biomarkers of proarrhythmia, Langendorff perfused rabbit heart, repolarization reserve, torsades de pointes

(J Cardiovasc Pharmacol ${ }^{\mathrm{TM}}$ 2014;64:266-276)

\section{INTRODUCTION}

Drug-induced torsades de pointes (TdP) is a lifethreatening arrhythmia. Preclinical drug development requires specific and sensitive surrogate biomarkers of TdP. ${ }^{1}$ To date, still the electrocardiogram (ECG) QT and heart rate corrected QT (QTc) intervals are the most accepted TdP surrogates by the authorities. ${ }^{2,3}$ However, the predictive power of these parameters has been questioned, ${ }^{4-7}$ therefore better predictors of TdP are needed.

Temporal inhomogeneity of repolarization has been reported to predict TdP development. ${ }^{6,8}$ Investigators prefer measuring repolarization inhomogeneity in regular (arrhythmia-free) rhythm because beat-to-beat variability of the repolarization in regular rhythm predicted TdP in earlier investigations. ${ }^{9,10}$ However, the same variables measured in regular rhythm could not forecast drug-induced TdP in other investigations. ${ }^{4,5,7,11}$ One of the possible reasons for this controversy is that $\mathrm{TdP}$ is frequently preceded by simple, drug-induced arrhythmias, which preclude ECG interval measurement in regular rhythm. To overcome this problem, we developed a method to allow ECG intervals to be measured during disorganized nonsinus rhythm. ${ }^{5,12}$ This allowed established beat-to-beat variability parameters to be derived irrespective of rhythm. ${ }^{5}$ To differentiate from published beat-to-beat variability parameters derived during arrhythmia-free rhythm (sinus beat-to-beat variability parameters), we coined the term "absolute" to describe the beat-to-beat variability parameters derived irrespective of rhythm. ${ }^{5}$ Absolute beat-to-beat variability parameters of the ECG intervals accurately predicted druginduced $\mathrm{TdP}$ and ischemia-induced ventricular fibrillation (VF), whereas equivalent variables measured in sinus rhythm failed to predict TdP and VF liability. ${ }^{5,13}$

In human and in many mammals (eg, guinea pig, rabbit, and dog), a considerable repolarizing current is the rapid component of the delayed rectifier $\mathrm{K}^{+}$current $\left(\mathrm{I}_{\mathrm{Kr}}\right)$, which is a common target of proarrhythmic drugs. However, under normal conditions, $\mathrm{I}_{\mathrm{Kr}}$ block just rarely evokes $\mathrm{TdP}$ as other repolarizing currents, eg, the slow component of the delayed rectifier 
$\mathrm{K}^{+}$current $\left(\mathrm{I}_{\mathrm{Ks}}\right.$ ), become more prominent and decrease the arrhythmogenic effect of the $\mathrm{I}_{\mathrm{Kr}}$ blocker. ${ }^{14,15}$ In contrast, the concomitant block of $\mathrm{I}_{\mathrm{Kr}}$ and $\mathrm{I}_{\mathrm{Ks}}$ currents reduces repolarization reserve ${ }^{16}$ and constitutes an increased risk to TdP development. ${ }^{17}$

We hypothesized that isolated hearts with pharmacologically reduced repolarization reserve would be suitable for preclinical assessment of the proarrhythmic liability of drugs. However, it is not known which proarrhythmia biomarker indicates the increased proarrhythmic risk in such experimental setting. Thus, the aim of our study was to investigate which proarrhythmia biomarker (QTc, sinus or absolute beatto-beat variability of the QT interval) can adequately indicate the increased proarrhythmic liability in the setting of reduced repolarization reserve in an in vitro, spontaneously beating rabbit heart proarrhythmia model. Reduced repolarization reserve was achieved by concomitant pharmacological inhibition of $\mathrm{I}_{\mathrm{Kr}}$ and $\mathrm{I}_{\mathrm{Ks}}$.

\section{METHODS}

\section{Animals}

Female New Zealand white rabbits weighing 1.6-2.5 $\mathrm{kg}$ were used for the experiments. Animals were obtained from WOBE Ltd (Budapest, Hungary) and acclimatized at the site for at least 3-4 days before any experiments started. Animals were kept under standard conditions (temperature $21^{\circ} \mathrm{C}$; relative humidity $55 \%-65 \%$; 12:12-hour dark/light cycle) on commercial laboratory chow and tap water ad libitum. Animal maintenance and research were conducted in accordance with the National Institutes of Health Guide for the Care and Use of Laboratory Animals. All procedures using animals were approved by the local ethics committee (Ethical Committee for the Protection of Animals in Research at the University of Szeged, Hungary) and conformed to the rules and principles of the 86/609/EEC Directive.

\section{General Methods}

General experimental method was performed as described earlier. ${ }^{18}$ The animals were anticoagulated with sodium heparin (1000 IU) injected into the marginal ear vein and stunned by a blow to the neck. The heart was rapidly removed through thoracotomy and rinsed in ice-cold modified Krebs-Henseleit buffer solution containing (in millimole): $\mathrm{NaCl} 118.4$, glucose $10.1, \mathrm{MgSO}_{4} 0.5, \mathrm{NaH}_{2} \mathrm{PO}_{4} 1.2, \mathrm{KCl}$ $3, \mathrm{NaHCO}_{3} 25$, and $\mathrm{CaCl}_{2}$ 1.8. The aorta was cannulated and hung on a Langendorff apparatus. The hearts were retrogradely perfused at a constant temperature of $37^{\circ} \mathrm{C}$ with the modified Krebs-Henseleit buffer solution described above. A mixture of $95 \% \mathrm{O}_{2}$ and $5 \% \mathrm{CO}_{2}$ was bubbled through the buffer, which was equilibrated to $\mathrm{pH}$ 7.4. All solutions were filtered ( $5 \mu \mathrm{m}$ pore size filter) before use. The perfusion pressure was maintained constant at $80 \mathrm{~mm} \mathrm{Hg}$. Volumeconducted ECG was recorded by using National Instruments data acquisition hardware (PC card; National Instruments, Austin, TX) and SPEL Advanced Haemosys software (version 2.76; Experimetria Ltd and Logirex Software Laboratory, Budapest, Hungary). Coronary flow was measured by timed collection of coronary effluent. Hearts were equilibrated for 15 minutes before starting the experimental protocol. At the end of each experiment, the atria were removed from the heart and the ventricles were weighed.

\section{Experimental Protocol}

In the first set of experiments, 3 groups of hearts were compared: (1) control group of hearts perfused with dimethyl sulphoxide (DMSO), that is the solvent of dofetilide and HMR-1556 ("Control A" group; $\mathrm{n}=6$ ), (2) hearts perfused with dofetilide at $15 \mathrm{nM}$ ("Dof 15" group; $\mathrm{n}=8$ ), and (3) hearts perfused with HMR-1556 at $460 \mathrm{nM}+$ dofetilide at 15 nM ("HMR + Dof 15" group; $\mathrm{n}=6)($ Table 1).

In the first set of experiments, there were only few druginduced arrhythmias; therefore, we performed a second set of experiments, in which we intended to increase the incidence of arrhythmias. In the second set of experiments, 4 groups (each contained 8 hearts) were compared: (1) control group of hearts perfused with DMSO and water, the latter is the solvent of catecholamines ("Control B" group), (2) hearts perfused with catecholamines (Cat) (adrenaline $25 \mathrm{nM}+$ noradrenaline $100 \mathrm{nM}$ ) + DMSO ("Cat Control" group), (3) hearts perfused with catecholamines + dofetilide at $50 \mathrm{nM}$ ("Cat + Dof 50" group), and (4) hearts perfused with catecholamines + HMR1556 at $460 \mathrm{nM}+$ dofetilide at $50 \mathrm{nM}$ ("Cat + HMR + Dof 50 " group) (Table 2).

The administration of the $\mathrm{I}_{\mathrm{Ks}}$ inhibitor HMR-1556 or its solvent (DMSO) was started at the beginning of a 30-minute pretreatment period. Dofetilide or its solvent (DMSO) was added to the perfusion solution after the pretreatment period, at the beginning of a 30-minute treatment period. In the second set of experiments, catecholamines or their solvent were administered from the beginning of the pretreatment period (Tables 1 and 2).

In the first set of experiments, we aimed to achieve a sufficient impairment of the repolarization without provoking a prominent arrhythmic activity. Thus, the selective $\mathrm{I}_{\mathrm{Kr}}$ blocker dofetilide was applied at a concentration of $15 \mathrm{nM}$, which inhibited approximately $50 \%$ of the $\mathrm{I}_{\mathrm{Kr}} \mathrm{K}^{+}$ current $^{19,20}$ and led to QTc prolongation without significant arrhythmic activity in our pilot study.

In the second set of experiments, we intended to increase the incidence of arrhythmias by further repolarization impairment. The concentration of dofetilide was increased to $50 \mathrm{nM}$ to achieve complete inhibition of $\mathrm{I}_{\mathrm{Kr}}$. Because $\mathrm{I}_{\mathrm{Ks}}$ blockade did not significantly increase repolarization instability unless $\beta$-adrenergic stimulation was added in canines in vivo, ${ }^{21,22}$ catecholamines were added to the perfusion solution to mimic the

TABLE 1. Experimental Protocol in the First Set of Experiments

\begin{tabular}{lcc}
\hline & \multicolumn{2}{c}{ Period } \\
\cline { 2 - 3 } Group & $\begin{array}{c}\text { Pretreatment } \\
\mathbf{( 3 0} \text { min) }\end{array}$ & $\begin{array}{c}\text { Treatment } \\
\mathbf{( 3 0} \text { min) }\end{array}$ \\
\hline Control A $(\mathrm{n}=6)$ & Vehicle & Vehicle \\
Dof 15 $(\mathrm{n}=8)$ & Vehicle & Dofetilide 15 $\mathrm{nM}$ \\
HMR + Dof 15 $(\mathrm{n}=6)$ & HMR & HMR + Dofetilide 15 $\mathrm{nM}$ \\
\hline
\end{tabular}

"Dof 15", group perfused with $15 \mathrm{nM}$ dofetilide; "HMR + Dof 15", group perfused with $460 \mathrm{nM}$ HMR-1556 and $15 \mathrm{nM}$ dofetilide; HMR, HMR-1556 at $460 \mathrm{nM}$; vehicle, DMSO (dimethyl sulphoxide), the common solvent of the dofetilide and HMR-1556. 
TABLE 2. Experimental Protocol in the Second Set of Experiments

\begin{tabular}{|c|c|c|}
\hline \multirow[b]{2}{*}{ Group } & \multicolumn{2}{|r|}{ Period } \\
\hline & $\begin{array}{l}\text { Pretreatment } \\
\text { (30 min) }\end{array}$ & $\begin{array}{l}\text { Treatment } \\
(30 \text { min) }\end{array}$ \\
\hline Control B $(\mathrm{n}=8)$ & Vehicles & Vehicles \\
\hline Cat control $(n=8)$ & Cat. + vehicle & Cat. + vehicle \\
\hline Cat + Dof $50(n=8)$ & Cat. + vehicle & Cat. + Dofetilide $50 \mathrm{nM}$ \\
\hline $\begin{array}{l}\text { Cat + HMR + Dof } 50 \\
\quad(n=8)\end{array}$ & Cat. + HMR & Cat. + HMR + Dofetilide $50 \mathrm{nM}$ \\
\hline
\end{tabular}

Vehicles in the "Control B" group are water acidified with ascorbic acid (the solven of the catecholamines) and DMSO (the common solvent of the dofetilide and HMR1556). Vehicle in the other groups is only DMSO. For further details see Table 1.

"Cat + Dof 50", group perfused with catecholamines and $50 \mathrm{nM}$ dofetilide; "Cat + HMR + Dof 50", group perfused with catecholamines, $460 \mathrm{nM}$ HMR-1556 and $50 \mathrm{nM}$ dofetilide; Cat., catecholamines (adrenaline $25 \mathrm{nM}+$ noradrenaline $100 \mathrm{nM}$ ).

sympathetic activity and to boost the function of $\mathrm{I}_{\mathrm{Ks}}$ (and the effect of $\mathrm{I}_{\mathrm{Ks}}$ inhibition) in the isolated rabbit hearts.

Because the $\mathrm{I}_{\mathrm{Ks}}$ inhibitor HMR-1556 may adsorb to glass or plastic surfaces, leading to reduced effective drug concentrations in the heart, the concentration of HMR-1556 was measured in the perfusate right above the heart by the means of spectrophotometry (Hitachi F-4000; Hitachi Ltd, Tokyo, Japan) with an excitation and emission wavelength of 295 and $325 \mathrm{~nm}$, respectively (data not shown). Because the $\mathrm{IC}_{50}$ of HMR-1556 ranged between $10.5^{23}$ and $65 \mathrm{nM},{ }^{17}$ our target concentration of HMR-1556 was $250 \mathrm{nM}$ to achieve a significant inhibition of the $\mathrm{I}_{\mathrm{Ks}}$. However, it was found in the pilot experiments that the concentration of HMR-1556 in the perfusate was lower at the level of the aorta than in the working buffer. Thus, we increased the concentration of HMR-1556 to $460 \mathrm{nM}$ in the working buffer, which resulted in a stable concentration of approximately $250 \mathrm{nM}$ HMR-1556 in the perfusate at the level of the hearts in our perfusion setup.

Experiments were randomized, and blinded analysis was achieved by using stock solutions prepared by a second operator, who did not participate in the experimental work or data analysis.

\section{Drugs and Materials}

Perfusion solutions were prepared fresh each day. Dofetilide and HMR-1556 were synthesized at Gedeon Richter Plc (Budapest, Hungary) and were dissolved in DMSO. All final test solutions for heart perfusion contained $0.045 \mathrm{~mL}$ DMSO in $1 \mathrm{~L}$ of modified Krebs-Henseleit solution. Catecholamines (adrenaline and noradrenaline) were purchased from Sigma Aldrich, Inc (St. Louis, MO) and were dissolved in water acidified with ascorbic acid; all final test solutions for heart perfusion contained $0.2 \mathrm{~mL}$ water and 25 $\mu$ mole ascorbic acid in $1 \mathrm{~L}$ of modified Krebs-Henseleit solution in the second set of experiments.

Chemicals for modified Krebs-Henseleit physiological solution were purchased from Reanal Private Ltd (Budapest, Hungary). DMSO and ascorbic acid were obtained from Sigma-Aldrich, Inc. Water for the preparation of perfusion solution was obtained from a reverse osmosis system (Milli-Q RG; Millipore Ltd, Billerica, MA) fed by distilled water and had a specific resistivity of $>18 \mathrm{M} \Omega$.

\section{Exclusion Criteria}

Any heart with a sinus rate $<120 /$ min or a coronary flow $>10 \mathrm{~mL} \cdot \mathrm{min}^{-1} \cdot \mathrm{g}^{-1}$ or $<3 \mathrm{~mL} \cdot \mathrm{min}^{-1} \cdot \mathrm{g}^{-1} 5$ minutes before the start of the 60 -minute drug perfusion protocol or not in a constant sinus rhythm before the start of the 60minute drug perfusion, was excluded. The excluded hearts were replaced to maintain equal group sizes when they may influence the main result.

\section{Arrhythmia Diagnosis}

The incidence and the time to onset of arrhythmias were determined. Ventricular premature beat (VPB), bigeminy, salvo, ventricular tachycardia (VT), and VF were defined according to the Lambeth Conventions. ${ }^{24} \mathrm{TdP}$ was defined as a polymorphic VT consisting of 4 or more ventricular complexes where clear twisting of the QRS complexes around the isoelectric axis could be seen in at least 1 ECG lead. ${ }^{12}$ Runs of 4 or more VPBs without the TdP-like twisting QRS morphology were differentiated from TdP and were defined as VT. Blocks in the conduction system were also monitored because blocks frequently occur in rabbits treated with repolarization prolonging drugs. ${ }^{25}$ Conduction disturbances included atrioventricular blocks and intraventricular conduction defects (right or left bundle branch blocks). ${ }^{5}$

\section{Measurement of the ECG Intervals and QTC}

ECG intervals were measured in sinus rhythm at predetermined time points $(14,25,35,44,50$, and 55 minutes). After the completion of experiments, the data were replayed with the SPEL Advanced Haemosys software (version 2.76; Experimetria Ltd and Logirex Software Laboratory). The software averaged the ECG signal from 20 consecutive sinus beats at the predetermined time point. If there were not 20 consecutive sinus beats at the predetermined time point or in the preceding or subsequent 30 seconds, then the measurement was not performed at the time point. The RR and QT intervals were measured by manual positioning on screen markers in the signal-averaged ECG. Heart rate was calculated from the RR interval. The QT interval was defined as the time from the first deviation from the isoelectric line during the PR interval until the end of the $\mathrm{T}$ wave. Where the $\mathrm{T}$ wave overlapped the following $\mathrm{P}$ wave or the QRS complex of the subsequent beat, the extrapolation method was used to measure the length of the QT interval, that is, the end of the $\mathrm{T}$ wave was extrapolated from the curve of the $\mathrm{T}$ wave to the isoelectric line under the $\mathrm{P}$ wave or the QRS complex. ${ }^{25}$

The QT interval is influenced by the heart rate, thus rate-corrected QT interval (QTc) was calculated with a correction method described earlier. ${ }^{26,27}$ Baseline data in sinus rhythm for QT intervals together with the corresponding RR intervals were obtained from pooled data of our laboratory (from 100 isolated, Langendorff-perfused rabbit hearts prepared as described above). The linear regression between the QT interval and the preceding RR interval was: $\mathrm{QT}_{\mathrm{n}+1}=$ $0.34 \mathrm{RR}_{\mathrm{n}}+90$ (Fig. 1A). This equation was rearranged to allow the calculation of the rate-corrected QT interval at a mean RR interval of 340 milliseconds (ie, a ventricular rate of approximately 177 beats per minute) using the formula 
$\mathrm{QTc}_{\mathrm{n}+1}=\mathrm{QT}_{\mathrm{n}+1}-0.34\left(\mathrm{RR}_{\mathrm{n}}-340\right)$. With these equations, plotting QTc against the corresponding RR interval produces a regression line with a slope of zero (Fig. 1B), indicating that these corrections successfully removed the influence of heart rate.

\section{Measurement of the Absolute and Sinus Beat- to-beat Variability of the QT and RR Interval}

To determine beat-to-beat variability of the RR and QT intervals, all analyses were based on samples of 40 consecutive ventricular complexes (RR intervals) at the predetermined time points, that is at baseline ( 1 minute before the pretreatment period), in the last minute of the pretreatment period, and in the fifth minute of the treatment period. From these samples, each of the $40 \mathrm{RR}$ and QT intervals was
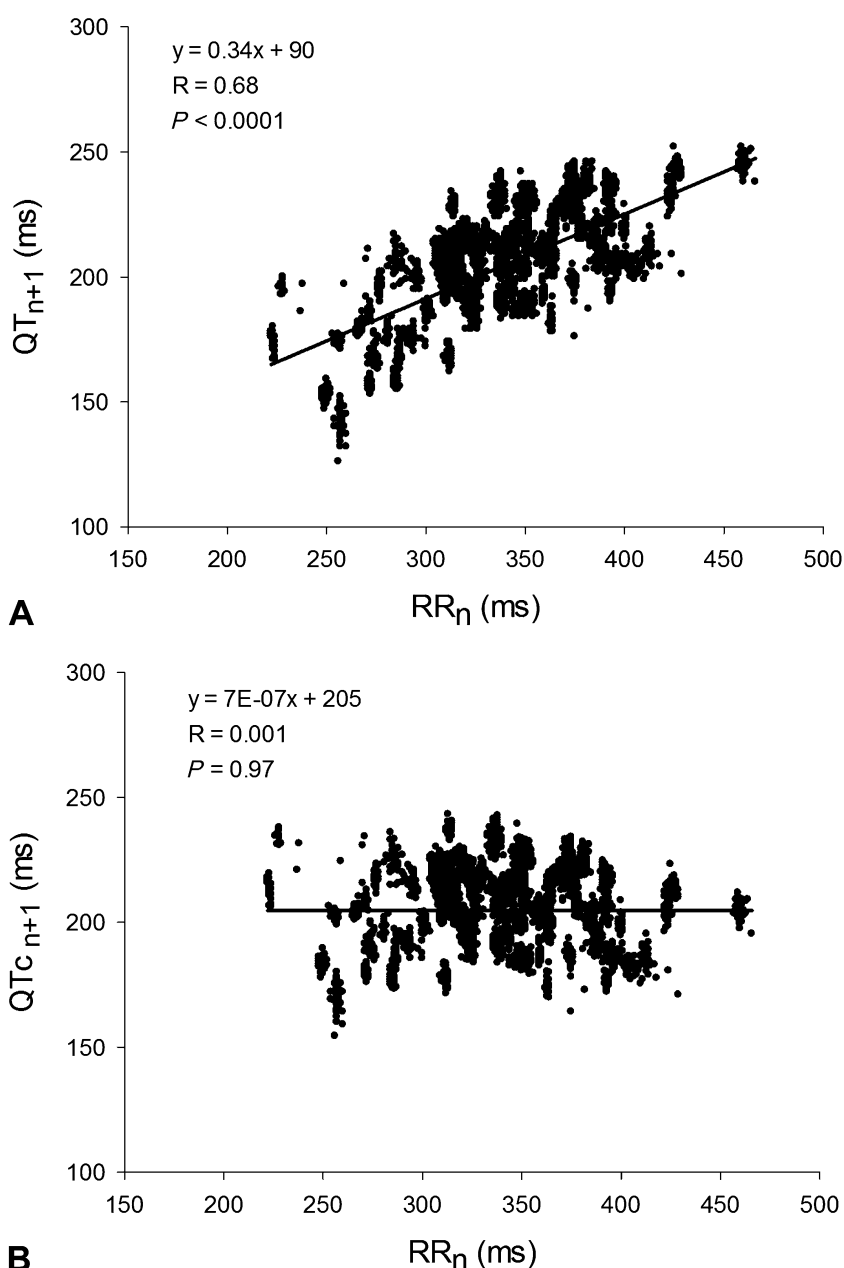

FIGURE 1. A, Correlation between individual values of the $\mathrm{QT}_{\mathrm{n}+1}$ interval and the preceding $\mathrm{RR}\left(\mathrm{RR}_{\mathrm{n}}\right)$ interval in sinus rhythm in isolated Langendorff-perfused rabbit hearts. $B$, Correlation between individual values of the "rate corrected" $\mathrm{QT}_{\mathrm{n}+1}\left(\mathrm{QTC}_{\mathrm{n}+1}\right)$ interval and the preceding $\mathrm{RR}\left(\mathrm{RR}_{\mathrm{n}}\right)$ interval in sinus rhythm in isolated Langendorff-perfused rabbit hearts. QTc was calculated using the formula $\mathrm{QTC}_{\mathrm{n}+1}=\mathrm{QT}_{\mathrm{n}+1-}$ $0.34\left(R_{n}-340\right)$. All panels contain 3900 baseline data points obtained from 100 isolated rabbit hearts. measured manually, then the short-term variability (STV) and the long-term variability (LTV) of the RR and QT intervals were derived as follows.

\section{Short-term Variability}

In terms of a Poincaré plot, which is a plot of the value of an ECG interval $\left(d_{i+1}\right)$ against the preceding value $\left(d_{i}\right)$, one can visualize the STV as the mean perpendicular distance between the points of the plot and the $d_{i+1}=d_{i}$ line. This corresponds to the following formula as described by Thomsen et $\mathrm{al}^{8}: \mathrm{STV}=\frac{1}{N \sqrt{2}} \sum_{i=0}^{N-2}\left(d_{i+1}-d_{i}\right)$, where $d_{i}$ represents the sequence of the ECG interval durations and $N$ is the total number of intervals.

\section{Long-term Variability}

In the framework outlined above, LTV is the mean distance (measured parallel to the $d_{i+1}=d_{i}$ line in the Poincaré plot) between the individual interval durations $\left(d_{i}\right)$ and their mean value $(E(d))$ as described by Thomsen et $\mathrm{al}^{8}$ : $\mathrm{LTV}=\frac{1}{N \sqrt{2}} \sum_{i=0}^{N-2}\left(d_{i+1}+d_{i}-2 E(d)\right)$.

The derived beat-to-beat variability parameters described above are referred to absolute beat-to-beat variability parameters when the raw ECG interval data were taken irrespective of the rhythm ie, regardless of whether rhythm was sinus or nonsinus at the time point of the measurement. The derived beat-to-beat variability parameters described above are referred to sinus beat-to-beat variability parameters when the raw ECG interval data were taken from samples of 40 consecutive complexes strictly in sinus rhythm at the predetermined time point. The sample of 40 consecutive sinus complexes was measured only if it was preceded by at least 10 arrhythmia-free beats to avoid any effect of preceding arrhythmias on the ECG intervals.

In the first set of experiments, arrhythmias were infrequent at the predetermined time points; therefore the measurement of the ECG intervals could be done in stable sinus rhythm. Thus, only sinus beat-to-beat variability parameters were determined at all of the predetermined time points. However, in the second set of experiments, hearts usually experienced arrhythmias during drug perfusion at the predetermined time points. Thus, the measurement of the ECG intervals in sinus rhythm must have been done before the predetermined time point; if there were not 50 consecutive sinus beats at the predetermined time point or in the preceding 2 minutes, then the measurement was not performed, and sinus beat-to-beat variability parameters were not determined at the time point.

The percent frequency of arrhythmic beats (defined as VPBs or individual QRT complexes in a run of a salvo or VT) was calculated as number per 40 beats 100 times in each sample used for measuring absolute beat-to-beat variability of the ECG intervals in the second set of the experiments.

\section{Statistics}

Continuous data were expressed as mean \pm SEM. All data from independent samples, except arrhythmia incidences, were compared with Kruskal-Wallis tests. The incidences of 
arrhythmias were compared by using Fisher's exact probability test with the Bonferroni correction, ie, the $P$ values of Fisher's exact probability test were multiplied by the number of comparisons between the groups to allow multiple comparisons. ${ }^{28}$ $P<0.05$ was taken as indicative of a statistically significant difference between values.

\section{RESULTS}

\section{Arrhythmia Incidences, Frequency, and Onset Time of Arrhythmias}

\section{First Set of Experiments}

Hearts were in sinus rhythm during the "pretreatment" period; HMR-1556 (without dofetilide) did not evoke arrhythmias. Dofetilide tended to increase arrhythmia incidences in the Dof 15 and HMR + Dof 15 groups in the "treatment" period, but there were no significant differences in the arrhythmia incidences among the groups (data not shown). Dofetilide induced TdP in 1 heart $(13 \%)$ in the Dof 15 group; the drug did not evoke VF. There were no significant differences in the onset times of arrhythmias between the groups (data not shown).

\section{Second Set of Experiments}

As expected, catecholamines increased arrhythmic activity (Fig. 2). In the pretreatment period, catecholamine perfusion evoked mostly VPBs, the incidences of other types of arrhythmias (bigeminy, salvo, and VT) were low. HMR1556 on top of catecholamines did not increase the incidence of any arrhythmias; there were no significant differences in the incidences of arrhythmias between the groups (data not shown). TdP and VF did not occur in this period.

In the treatment period, addition of dofetilide on top of HMR-1556 and catecholamines significantly increased the frequency of arrhythmic beats as compared with control (Figs. 2, 3). However, there were no significant differences in the incidences of VPB, BG, and salvo among the groups (data

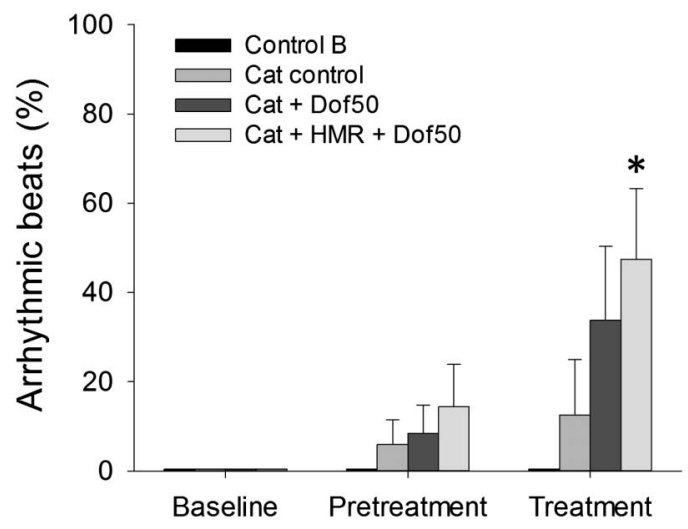

FIGURE 2. Percent frequency of arrhythmic beats in 40 consecutive ventricular beats at baseline (baseline), in the last minute of the pretreatment period (pretreatment), and 5 minutes after the start of the dofetilide (or its vehicle) perfusion (treatment) in the second set of experiments. $* P<0.05$ versus Control B group. For further details, see Table 2. not shown). TdP did not occur in the control groups, but dofetilide induced TdP in 1 heart in both dofetilide-perfused groups. Dofetilide coperfused with HMR-1556 and catecholamines tended to increase VT and VF incidences, but there were no significant differences in the incidences of VT and VF among the groups (VT: $0 \%, 25 \%, 38 \%$, and $63 \%$; VF: $0 \%, 0 \%, 25 \%$, and $38 \%$ in the Control B, Cat control, Cat + Dof 50, and Cat + HMR + Dof 50 groups, respectively). Coperfusion of dofetilide, HMR-1556, and catecholamines significantly increased the incidence of conduction blocks as an indirect sign of markedly prolonged repolarization [block incidences: $0 \%, 0 \%, 50 \%$, and 75\% $(P<0.05$ vs. Control B and Cat control) in Control B, Cat control, Cat + Dof 50, and Cat + HMR + Dof 50 groups, respectively]. There were no significant differences in the onset times of arrhythmias between the groups (data not shown).

\section{QTc Interval and Heart Rate in Sinus Rhythm}

\section{First Set of Experiments}

QTe did not differ between the groups in the pretreatment period (data not shown). In the treatment period, administration of dofetilide for 10 minutes significantly prolonged the QTc interval; however, QTc did not differentiate between the Dof 15 and the HMR + Dof 15 groups (Fig. 4A). Occurrence of dofetilide-induced arrhythmias did not allow measurement of the QTc interval after 10 minutes of dofetilide perfusion. There was no significant difference in the heart rate between the groups during the whole experiment (Fig. 5A).

\section{Second Set of Experiments}

QTc did not differ between the groups at baseline and in the pretreatment period, before dofetilide perfusion (data not shown). In the treatment period, administration of dofetilide for 10 minutes significantly widened the QTc interval; however, QTc did not differentiate between the Cat + Dof 50 and the Cat + HMR + Dof 50 groups (Fig. 4B); dofetilideinduced arrhythmias prevented further measurements of the biomarker at the subsequent time points. Although catecholamines increased heart rate (Figs. 3, 5B), and HMR-1556 tended to cause bradycardia, there was no significant difference in the heart rate between the groups during the whole experiment (Fig. 5B).

\section{Temporal Inhomogeneity of the Repolarization and the Cycle Length}

\section{First Set of Experiments}

The STV and LTV parameters of the RR and QT intervals were determined in sinus rhythm (sinus variability parameters). The sinus STV QT and the sinus LTV QT parameters did not differ between the groups at baseline and during the pretreatment period (data not shown). In the treatment period, dofetilide perfusion for 5 minutes increased the sinus STV QT and the sinus LTV QT values. Importantly, both sinus STV QT and sinus LTV QT increased significantly in the HMR + Dof 15 group as compared with the values in the Dof 15 and the Control A groups (Fig. 6). The sinus variability parameters could not be determined at subsequent 

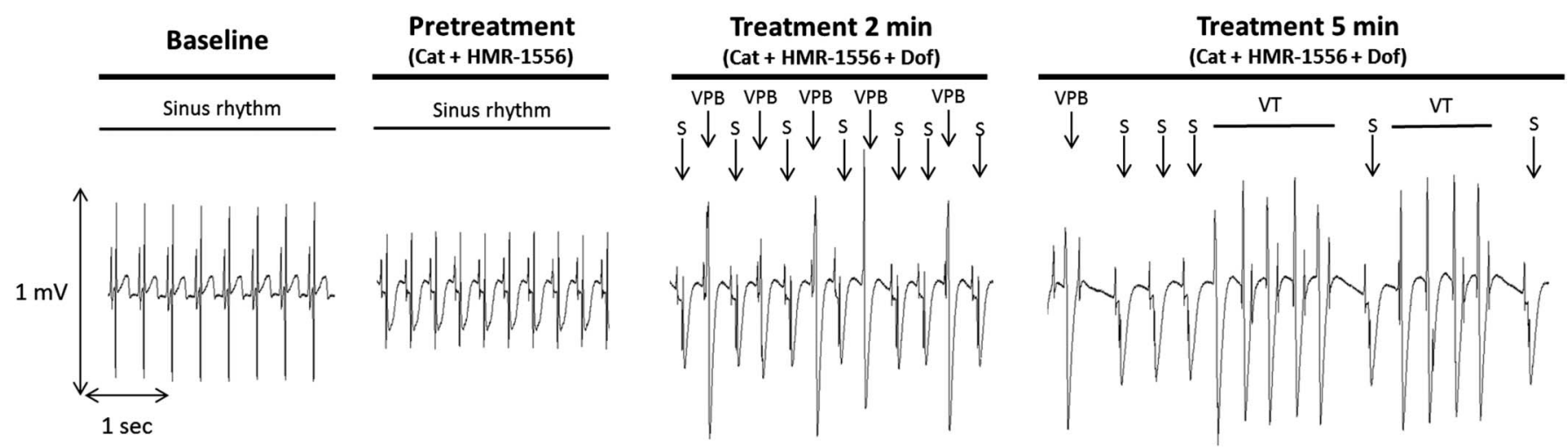

FIGURE 3. An example of development of dofetilide-induced arrhythmias when coperfused with HMR-1556 and catecholamines in a heart from the second set of experiments. ECG signals were recorded before drug administration (baseline), in the last minute of the pretreatment period (pretreatment), and 2 and 5 minutes after starting dofetilide perfusion (treatment 2 and treatment 5 minutes, respectively). S, sinus beat; VPB, ventricular premature beat; VT, ventricular tachycardia.

time points because of frequent occurrence of dofetilideinduced arrhythmias. There was no significant difference in any of the variability parameters of the RR interval at any time points between the groups (data not shown).

\section{Second Set of Experiments}

The STV and LTV of the RR and QT intervals were determined not only in sinus rhythm (sinus variability parameters) but also irrespective of the rhythm (absolute variability parameters).

\section{The Sinus Variability Parameters of the ECG Intervals}

The sinus variability parameters of the QT and RR intervals did not differ significantly between the groups at any predetermined time points (Figs. 7, 8). Neither catecholamines nor HMR-1556 influenced the sinus variability parameters during the pretreatment period (data not shown). Dofetilide coadministered with HMR-1556 for 5 minutes increased the sinus STV QT and LTV QT in the Cat + HMR + Dof 50 group as compared with the values of the $\mathrm{Cat}+\mathrm{Dof} 50$ and the control groups. However, the effect was not significant, most probably because the parameter could be determined only in 5 hearts in the Cat + HMR + Dof 50 group because of frequent occurrence of dofetilide-induced arrhythmias (Fig. 7). These arrhythmias did not allow measurement of the sinus variability parameters at subsequent time points.

\section{The Absolute Beat-to-beat Variability of the ECG Intervals}

Unlike sinus variability parameters, absolute variability parameters could be determined in all hearts because measurement of the absolute variability parameters is not restricted to sinus rhythm. None of the absolute variability parameters of the RR and QT intervals differed between the groups at baseline and during the pretreatment period (data not shown). In the treatment period, coperfusion of dofetilide with HMR-1556 for 5 minutes increased the absolute variability parameters of the QT and RR intervals (Figs. 7-9). Importantly, the absolute STV QT and the absolute LTV QT parameters differentiated between the Cat + Dof 50 and Cat + HMR + Dof 50 groups; that is, the absolute variability parameters of the QT interval were significantly increased in the Cat + $\mathrm{HMR}+$ Dof 50 group as compared with the values in the Cat + Dof 50 group (Fig. 7).

\section{Coronary Flow}

The mean baseline coronary flow values ranged from $4.1 \pm 0.3$ to $7.3 \pm 0.5 \mathrm{~mL} \cdot \mathrm{min}^{-1} \cdot \mathrm{g}^{-1}$ in the first set of experiments and from $4.2 \pm 0.3$ to $7.4 \pm 0.7 \mathrm{~mL} \cdot \mathrm{min}^{-1} \cdot \mathrm{g}^{-1}$ in the second set of experiments. There was a small timedependent fall in the coronary flow values during the experiments in all groups. In the first set of experiments, there was no significant difference in the coronary flow between the groups. In the second set of experiments, catecholamine perfusion
FIGURE 4. The QTc intervals $10 \mathrm{mi}$ nutes after the start of the dofetilide (or its vehicle) perfusion in the first set of experiments $(A)$ and in the second set of experiments (B). $\dagger P<$ 0.05 versus Control $A$ group; $* P<0.05$ versus Control B group; $\# P<0.05$ versus Cat control group. For further details, see Tables 1 and 2.
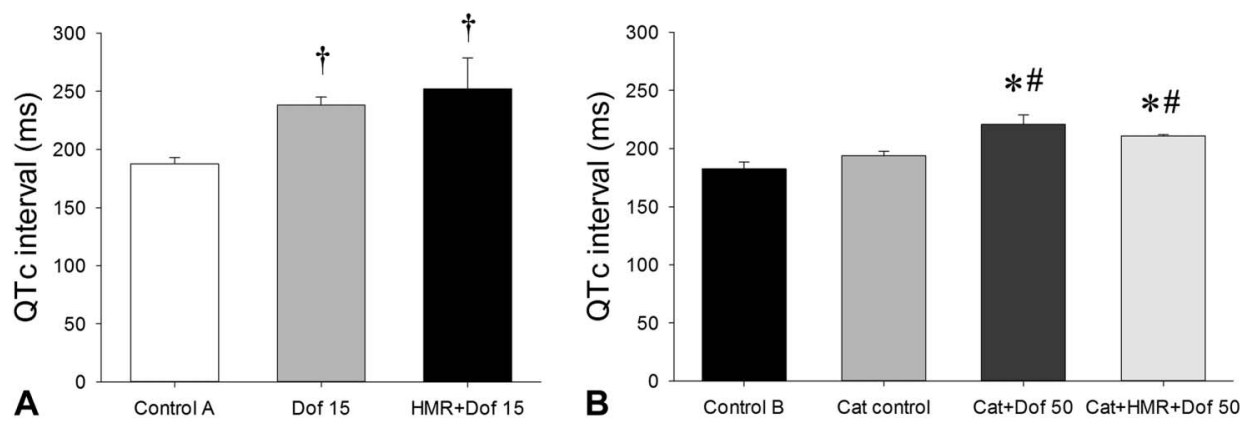
FIGURE 5. The heart rate in the first set of experiments $(A)$ and in the second set of experiments (B). HMR1556 (or its vehicle) perfusion commenced at 0 minute (pretreatment period). Catecholamine perfusion was applied only in the second set of experiments; catecholamine (or vehicle) perfusion commenced at 0 minute (pretreatment period). Dofetilide (or its vehicle) perfusion began at $30 \mathrm{mi}$ nutes (treatment period). For further details, see Tables 1 and 2 .
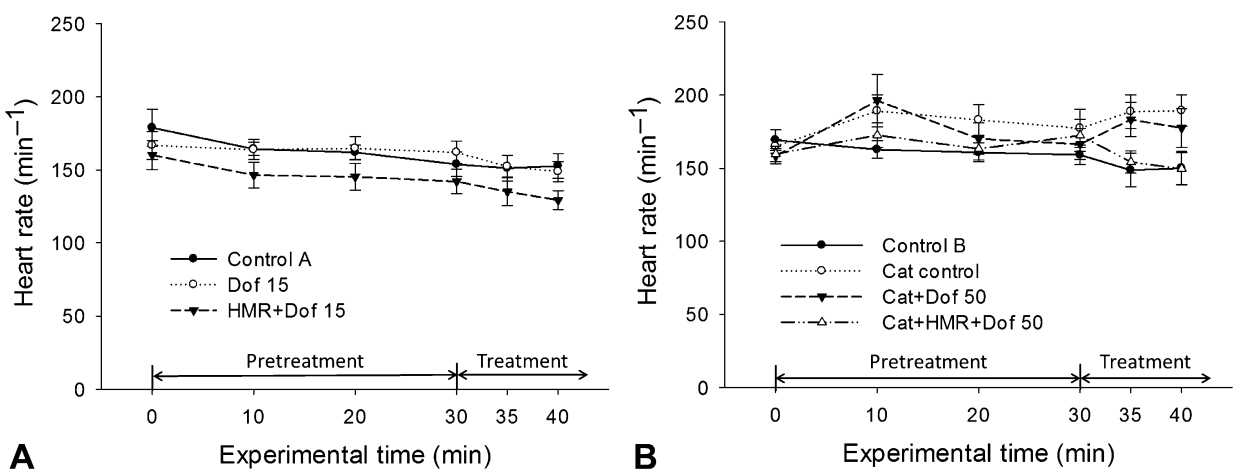

increased the flow slightly but there was no significant difference in the coronary flow between the groups (data not shown).

\section{DISCUSSION}

QTc failed to predict the known increased risk of proarrhythmia in the setting of reduced repolarization reserve. However, a significant elevation in the sinus beat-to-beat variability values of the QT interval (sinus STV QT and LTV QT) indicated the increased probability of proarrhythmia, when drug-induced arrhythmias were infrequent during administration of a low concentration of dofetilide. In contrast, when catecholamines and an elevated concentration of dofetilide was applied, sinus beat-to-beat variability parameters of the QT interval could not forecast an increased hazard to proarrhythmia occurrence because frequent druginduced arrhythmias precluded measurement of the beat-tobeat variability parameters in sinus rhythm. However, increased absolute beat-to-beat variability parameters of the QT interval (absolute STV QT and LTV QT), which are determined irrespective of the rhythm even during arrhythmias, were indicatives of the increased proarrhythmic liability in the setting of reduced repolarization reserve even when frequent arrhythmias compromised the measurement of the sinus STV QT and LTV QT.

\section{Decreased Repolarization Reserve Augments Risk of Proarrhythmia and Limits the Use of Biomarkers Restricted to Sinus Rhythm}

Based on the theory of repolarization reserve, the fine-tuned function of repolarization currents may compensate the lack of a repolarizing current, ${ }^{16} \mathrm{eg}, \mathrm{I}_{\mathrm{Ks}}$ may compensate the decreased function of $\mathrm{I}_{\mathrm{Kr}}$. However, a multiple hit on repolarization might lead to an excessive prolongation of the action potential duration (APD) and may render the cardiac muscle more susceptible to proarrhythmia. $^{9,29}$

Reduction of repolarization reserve increased the incidence of dofetilide-induced $\mathrm{TdP}$ in dogs and rabbits in vivo. ${ }^{9}$ Also, it was documented that the increased betaadrenergic tone augmented the effect of the $\mathrm{I}_{\mathrm{Ks}}$ inhibitor HMR-1556 on refractoriness observed at rapid rates in canines in vivo. ${ }^{21}$ Interestingly, neither increased concentration of dofetilide nor boosted HMR-1556-induced $\mathrm{I}_{\mathrm{Ks}}$ inhibition due to catecholamine administration could initiate significant amount of dofetilide-induced TdP in the present investigation in isolated rabbit hearts, which highlights the importance of the surrogate biomarkers of TdP in preclinical drug-safety investigations. The exact reason why a low incidence of TdP was observed in the present investigation is not examined. The incidence of TdP might have been increased with the application of higher concentrations of dofetilide and HMR-1556 (unpublished results from our laboratory); however, this was not the primary aim of this study. The combined $\mathrm{I}_{\mathrm{Ks}}$ and $\mathrm{I}_{\mathrm{Kr}}$ blockade did increase the frequency of less complex arrhythmias, and thus, prevented the use of proarrhythmia biomarkers that are based on ECG interval measurement restricted to sinus rhythm. However, the occurrence of arrhythmias provided scope for testing the predictive power of the novel proarrhythmia biomarkers, the absolute variability parameters ${ }^{5}$ that are not restricted to sinus rhythm.
FIGURE 6. The STV and the LTV of the QT interval 5 minutes after the start of the dofetilide (or its vehicle) perfusion in the first set of experiments. $* P<0.05$ versus Control A group; $\dagger P<0.05$ versus Dof 15 group. For further details, see Table 1.

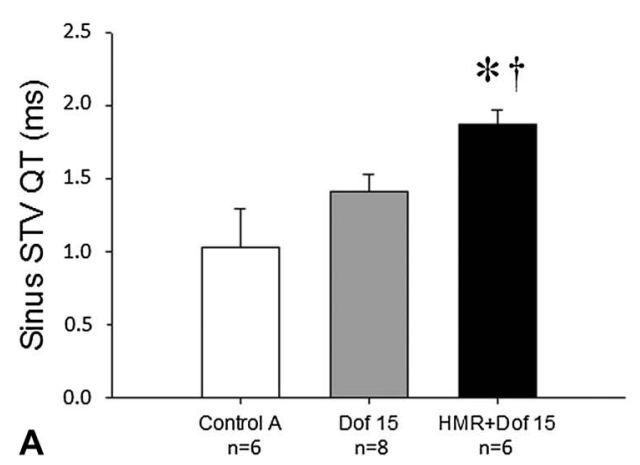

272 | www.jcvp.org

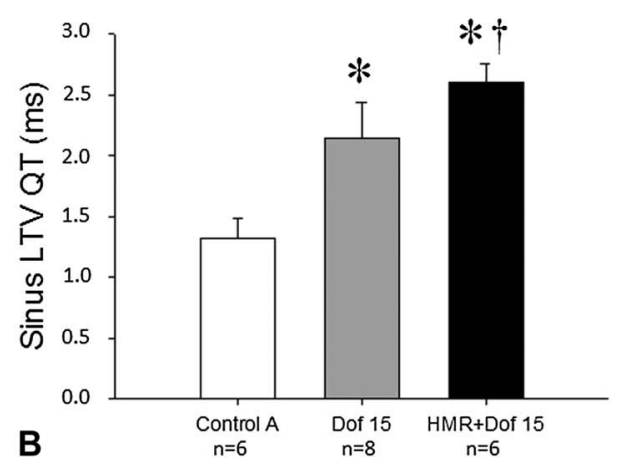

(C) 2014 Lippincott Williams \& Wilkins 

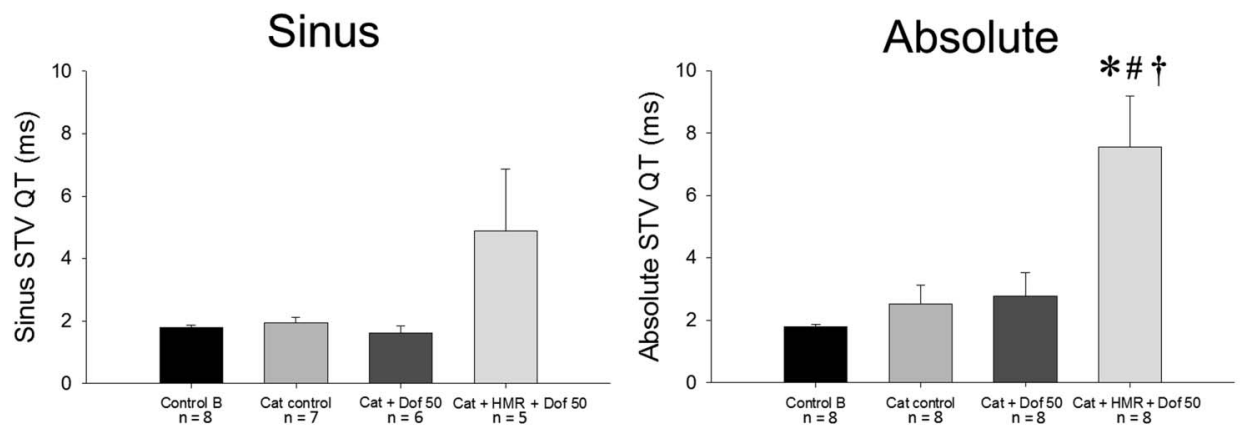

FIGURE 7. The sinus and absolute STV and LTV parameters of the QT interval in the treatment period, 5 minutes after the start of the dofetilide (or its vehicle) perfusion in the second set of experiments. $* P<0.05$ versus Control B group; $\# P<0.05$ versus Cat control group; $\dagger P<0.05$ versus Cat + Dof 50 group. For further details, see Table 2.
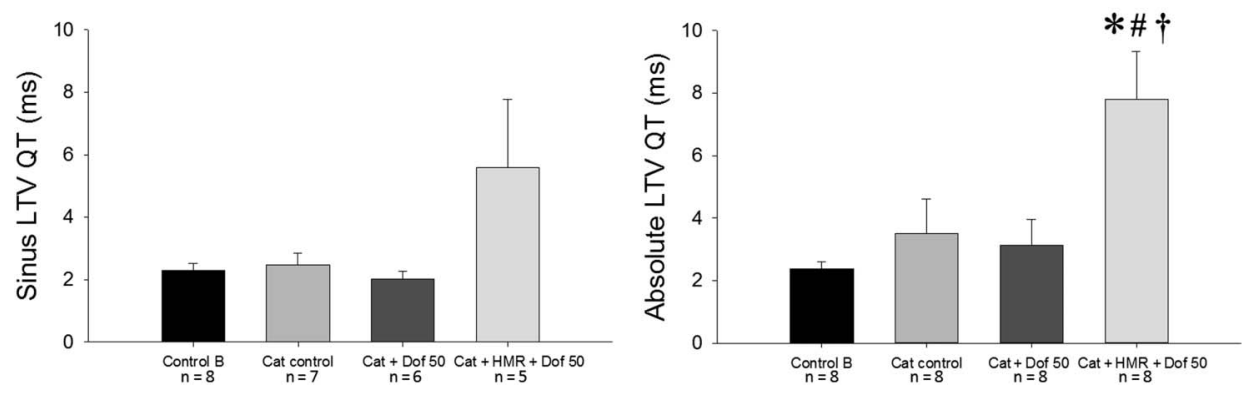

\section{QTc Failed to Predict Increased Proarrhythmia Risk in Reduced Repolarization Reserve}

In this study, the $\mathrm{I}_{\mathrm{Kr}}$ blocker dofetilide caused a significant QTc interval prolongation. However, the $\mathrm{I}_{\mathrm{Ks}}$ inhibitor HMR-1556 did not lengthen the QTc interval. Similarly, $\mathrm{I}_{\mathrm{Ks}}$ inhibition did not prolong the QTc interval in isolated rabbit hearts ${ }^{14}$ and did not lengthen the APD in cardiac papillary muscles ${ }^{14}$ reinforcing that $\mathrm{I}_{\mathrm{Ks}}$ may have little role in normal action potential repolarization and it probably plays a vital role when cardiac APD is abnormally lengthened by other means. ${ }^{29}$ Dofetilide alone and coperfused with the $\mathrm{I}_{\mathrm{Ks}}$ blocker HMR-1556 caused an equivalent degree of QTc prolongation, thus the extent of the QTc prolongation did not differ between the presence and absence of the $\mathrm{I}_{\mathrm{Ks}}$ inhibition. Similarly, Lengyel et $\mathrm{al}^{9}$ found that the concomitant inhibition of $\mathrm{I}_{\mathrm{Ks}}$ and $\mathrm{I}_{\mathrm{Kr}}$ with dofetilide and HMR-1556 did not prolong the QTc interval significantly as compared with that achieved by the $\mathrm{I}_{\mathrm{Kr}}$ inhibition in anaesthetized rabbits and dogs in vivo. These results emphasize that QTc interval is not a suitable biomarker to identify increased proarrhythmia liability in case of attenuated repolarization reserve.
FIGURE 8. The sinus and absolute STV and LTV parameters of the RR interval in the treatment period, 5 minutes after the start of the dofetilide (or its vehicle) perfusion in the second set of experiments. $* P<$ 0.05 versus Control B group; $\# P<$ 0.05 versus Cat control group; $\dagger P<0.05$ versus Cat + Dof 50 group. For further details, see Table 2.
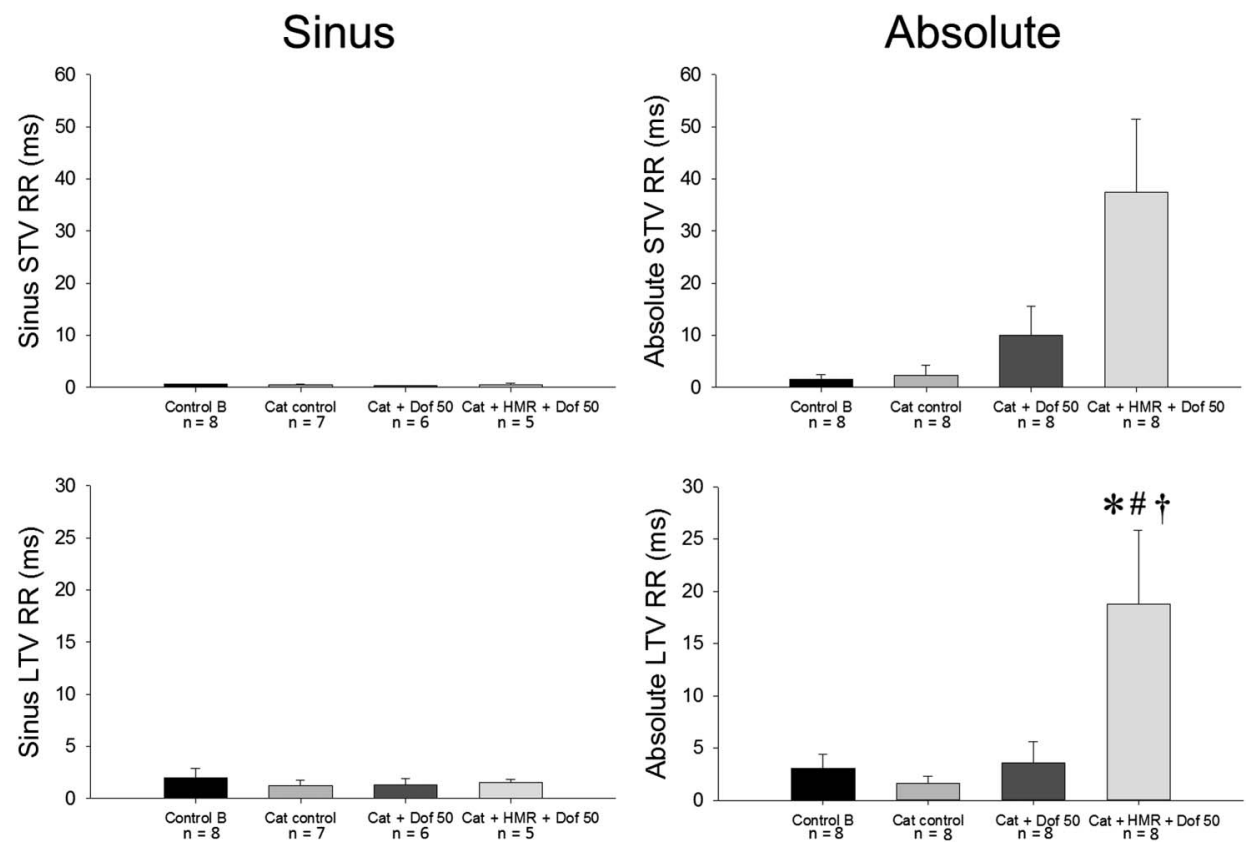


\section{Control B}
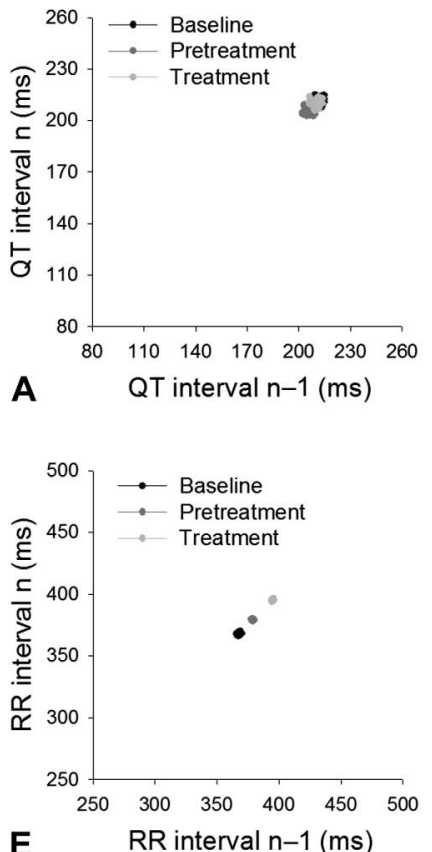

Cat control
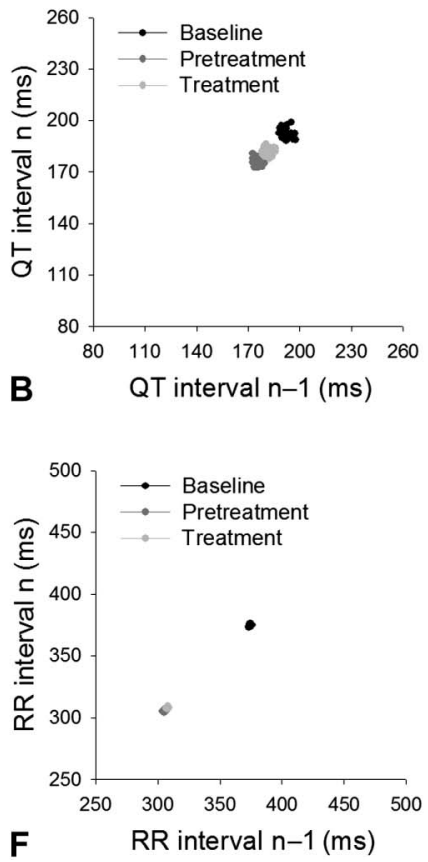

Cat + Dof 50
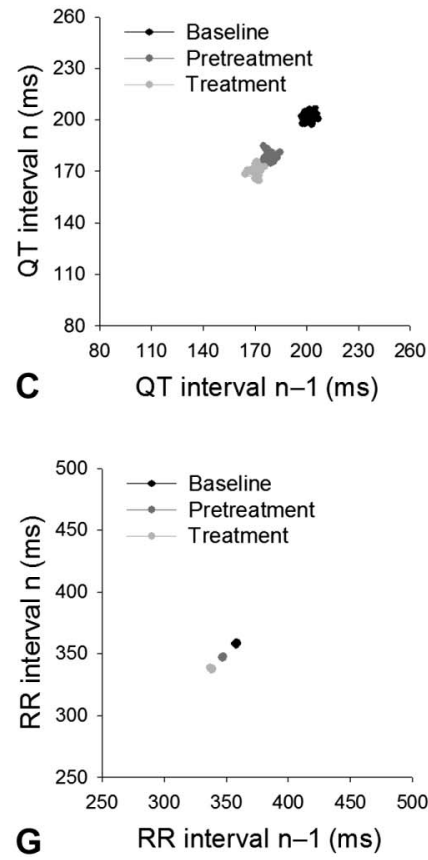

Cat + HMR + Dof 50
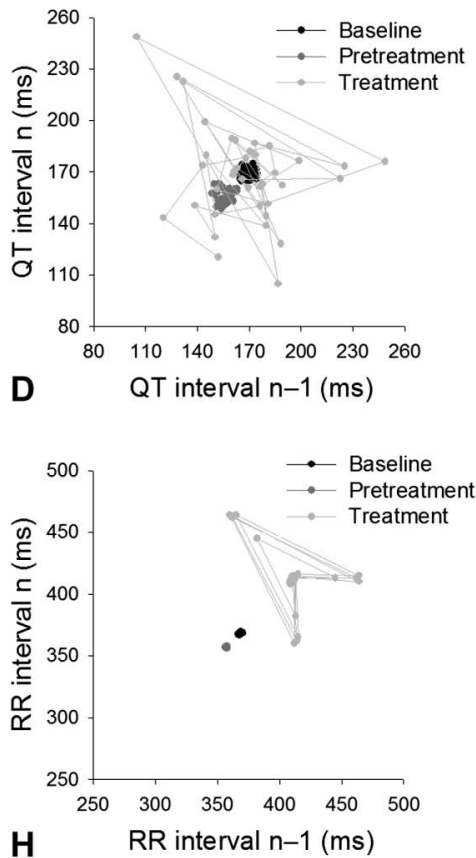

FIGURE 9. Poincaré plots of the QT and RR intervals measured irrespective of the rhythm in 1 representative experiment from Control $B$ group ( $A$ and $E$ ), in 1 representative experiment from Cat control group ( $B$ and $F$ ), in 1 representative experiment from Cat + Dof 50 group $(C$ and $G)$, and in 1 representative experiment from Cat + HMR + Dof 50 group (D and H). Values were measured at baseline (baseline), in the last minute of the pretreatment period (pretreatment) and 5 minutes after the start of the dofetilide (or its vehicle) perfusion (treatment) in the second set of experiments. For further details, see Table 2.

\section{Sinus Beat-to-beat Variability of the Repolarization is a Better Predictor of Proarrhythmia in Reduced Repolarization Reserve, but Arrhythmic Activity Limits Its Use}

It has been suggested that temporal dispersion of repolarization could predict proarrhythmic events. ${ }^{30,31}$ It was found in the chronic atrioventricular node-blocked dog model that a downregulation of several potassium currents, most notably the $\mathrm{I}_{\mathrm{Ks}}$ channel, occurs because of the electrical remodeling. ${ }^{32}$ Additional pharmacological block of $\mathrm{I}_{\mathrm{Kr}}$ did not prolong the QTc interval significantly in the dog model, but increased the beat-to-beat variability of the repolarization (STV QT), when it was measured in arrhythmia-free, regular rhythm. ${ }^{8}$ Lengyel et $\mathrm{al}^{9}$ measured STV QT in sinus rhythm in rabbits in vivo and directly demonstrated that TdP evoked by combined pharmacological block of $\mathrm{I}_{\mathrm{Kr}}$ and $\mathrm{I}_{\mathrm{Ks}}$ can be better predicted by increased STV QT than by QTc changes alone. Carlsson et $\mathrm{al}^{10}$ reported that dofetilide increased STV QT in sinus rhythm before the occurrence of VPBs and TdP in methoxamine-sensitized, anaesthetized rabbits. However, sinus STV QT did not predict TdP in other studies using a similar, anaesthetized rabbit model. ${ }^{4,5,7}$

In the first set of experiments in this investigation, the measurement of the beat-to-beat variability of the QT intervals was possible in sinus rhythm at the predetermined time points because the arrhythmic activity was low during concomitant pharmacological inhibition of $\mathrm{I}_{\mathrm{Ks}}$ and $\mathrm{I}_{\mathrm{Kr}}$. In the group perfused with dofetilide together with HMR-1556, the beat-to-beat variability parameters of the QT interval were significantly increased as compared with those in the control group, and more importantly, compared with those in the group perfused only with dofetilide. Accordingly, STV QT and LTV QT could differentiate the combined ion-channel block from the sole $\mathrm{I}_{\mathrm{Kr}}$ block identifying the increased proarrhythmia liability during decreased repolarization reserve.

However, in the second set of experiments, catecholamines and elevated concentration of dofetilide increased the arrhythmic activity, which precluded the measurement of the sinus beat-to-beat variability parameters of the QT intervals, thus these parameters failed to indicate the increased proarrhythmic liability of the drugs during reduced repolarization reserve. These results show that sinus beat-to-beat variability parameters of the repolarization (sinus STV QT and LTV QT) are better predictors of proarrhythmic liability than QTc in reduced repolarization reserve, but the application of these parameters is limited when arrhythmia occurs.

\section{Absolute Beat-to-beat Variability of the Repolarization Is the Best Proarrhythmia Predictor in Reduced Repolarization Reserve}

Dofetilide was used as a reference drug because it can cause TdP in humans and animal models., ${ }^{5,9,12,33}$ Earlier 
investigations showed that increased temporal variability of repolarization provides increased risk for development of drug-induced TdP. ${ }^{5,8}$ Dofetilide-induced temporal variability of repolarization measured by absolute STV QT and LTV QT increased markedly when the drug was coadministered with HMR-1556 and catecholamines in this investigation. This indicates that reduced repolarization reserve achieved by pharmacological inhibition of $\mathrm{I}_{\mathrm{Ks}}$ helps to reveal the proarrhythmic liability of $\mathrm{I}_{\mathrm{Kr}}$ inhibitors in isolated rabbit hearts.

In the presence of reduced repolarization reserve, ectopic beats can render the APD unstable for many beats ${ }^{6}$ and thus produce large beat-to-beat irregularity. ${ }^{34}$ Moreover, increased variability of repolarization may remain latent until the heart is disturbed by an ectopic beat. ${ }^{35}$ In a previous invivo study, most TdP events were preceded by noncomplex arrhythmias (eg, VPBs and salvos) in anesthetized rabbits. ${ }^{5}$ Similar noncomplex arrhythmias occurred frequently in the second set of experiments in the present in vitro investigation, when hearts were sensitized to arrhythmia development by the coperfusion of catecholamines and an elevated concentration of dofetilide. Because TdP rarely occurs spontaneously without preceding arrhythmias, however, the number of arrhythmic beats was not a precise predictor of druginduced $\mathrm{TdP},{ }^{5}$ new TdP biomarkers that can be measured irrespective of rhythm even during arrhythmias are needed. The predictive power of the sinus beat-to-beat variability of the repolarization is not consistent especially when frequent arrhythmias preclude its measurement. However, the newly developed absolute beat-to-beat variability parameters of the ECG intervals seem to be more reliable surrogate biomarkers of TdP. ${ }^{5}$ Indeed, in this study, where the sinus beat-to-beat variability was not predictive in the second set of experiments, the absolute beat-to-beat variability parameters of the repolarization still indicated the increased proarrhythmic liability of dofetilide in the setting of decreased repolarization reserve.

\section{CONCLUSIONS}

QTc prolongation did not identify the increased proarrhythmic risk of dofetilide in the setting of reduced repolarization reserve. In contrast, the sinus beat-to-beat variability of QT interval was a sensitive biomarker for proarrhythmia in the setting of decreased repolarization reserve. However, when measurement of the sinus variability parameters was compromised by arrhythmias, only absolute beat-to-beat variability parameters of the repolarization could reveal the increased proarrhythmic liability. Thus, absolute QT variability parameters seem to be the most practical and sensitive biomarkers of proarrhythmic liability in the setting of reduced repolarization reserve. Absolute STV QT and LTV QT could serve as surrogates for TdP in drug-safety investigations in isolated rabbit hearts with attenuated repolarization reserve.

\section{REFERENCES}

1. Farkas AS, Nattel S. Minimizing repolarization-related proarrhythmic risk in drug development and clinical practice. Drugs. 2010;70:573-603.

2. ICH. International Conference on Harmonisation; guidance on S7B nonclinical evaluation of the potential for delayed ventricular repolarization
(QT interval prolongation) by human pharmaceuticals; availability. Notice. Fed Regist. 2005;70:61133-61134.

3. ICH. International Conference on Harmonisation; guidance on E14 clinical evaluation of QT/QTc interval prolongation and proarrhythmic potential for non-antiarrhythmic drugs; availability. Notice. Fed Regist. 2005;70:61134-61135.

4. Farkas A, Dempster J, Coker SJ. Importance of vagally mediated bradycardia for the induction of torsade de pointes in an in vivo model. $\mathrm{Br} \mathrm{J}$ Pharmacol. 2008;154:958-970.

5. Farkas AS, Rudas L, Makra P, et al. Biomarkers and endogenous determinants of dofetilide-induced torsades de pointes in alpha(1)adrenoceptor-stimulated, anaesthetized rabbits. Br J Pharmacol. 2010; 161:1477-1495.

6. Hondeghem LM, Carlsson L, Duker G. Instability and triangulation of the action potential predict serious proarrhythmia, but action potential duration prolongation is antiarrhythmic. Circulation. 2001;103:20042013.

7. Vincze D, Farkas AS, Rudas L, et al. Relevance of anaesthesia for dofetilide-induced torsades de pointes in alpha ${ }_{1}$-adrenoceptor-stimulated rabbits. Br J Pharmacol. 2008;153:75-89.

8. Thomsen MB, Verduyn SC, Stengl M, et al. Increased short-term variability of repolarization predicts d-sotalol-induced torsades de pointes in dogs. Circulation. 2004;110:2453-2459.

9. Lengyel C, Varró A, Tábori K, et al. Combined pharmacological block of $\mathrm{I}(\mathrm{Kr})$ and $\mathrm{I}(\mathrm{Ks})$ increases short-term QT interval variability and provokes torsades de pointes. Br J Pharmacol. 2007;151:941-951.

10. Carlsson L, Andersson B, Linhardt G, et al. Assessment of the ion channel-blocking profile of the novel combined ion channel blocker AZD1305 and its proarrhythmic potential versus dofetilide in the methoxamine-sensitized rabbit in vivo. J Cardiovasc Pharmacol. 2009; 54:82-89.

11. Michael G, Kane KA, Coker SJ. Adrenaline reveals the torsadogenic effect of combined blockade of potassium channels in anaesthetized guinea pigs. $\mathrm{Br} J$ Pharmacol. 2008;154:1414-1426.

12. Farkas AS, Makra P, Csík N, et al. The role of the $\mathrm{Na}^{+} / \mathrm{Ca}^{2+}$ exchanger, I $(\mathrm{Na})$ and $\mathrm{I}(\mathrm{CaL})$ in the genesis of dofetilide-induced torsades de pointes in isolated, AV-blocked rabbit hearts. Br J Pharmacol. 2009;156:920-932.

13. Sarusi A, Rárosi F, Szücs M, et al. Absolute beat-to-beat variability and instability parameters of ECG intervals: biomarkers for predicting ischaemia-induced ventricular fibrillation. Br J Pharmacol. 2014;171: 1772-1782.

14. Lengyel C, Iost N, Virág L, et al. Pharmacological block of the slow component of the outward delayed rectifier current $(\mathrm{I}(\mathrm{Ks}))$ fails to lengthen rabbit ventricular muscle QT(c) and action potential duration. Br J Pharmacol. 2001;132:101-110.

15. Varró A, Baláti B, Iost $\mathrm{N}$, et al. The role of the delayed rectifier component $\mathrm{I}_{\mathrm{Ks}}$ in dog ventricular muscle and Purkinje fibre repolarization. $J$ Physiol. 2000;523(pt 1):67-81.

16. Roden DM. Taking the "idio" out of "idiosyncratic": predicting torsades de pointes. Pacing Clin Electrophysiol. 1998;21:1029-1034.

17. Volders PG, Stengl M, van Opstal JM, et al. Probing the contribution of $\mathrm{I}_{\mathrm{Ks}}$ to canine ventricular repolarization: key role for beta-adrenergic receptor stimulation. Circulation. 2003;107:2753-2760.

18. Farkas AS, Acsai K, Tóth A, et al. Importance of extracardiac alpha ${ }^{-}$ adrenoceptor stimulation in assisting dofetilide to induce torsade de pointes in rabbit hearts. Eur J Pharmacol. 2006;537:118-125.

19. Yang T, Roden DM. Extracellular potassium modulation of drug block of $\mathrm{I}_{\mathrm{Kr}}$. Implications for torsade de pointes and reverse use-dependence. Circulation. 1996;93:407-411.

20. Ollerstam A, Visser SA, Duker G, et al. Comparison of the QT interval response during sinus and paced rhythm in conscious and anesthetized beagle dogs. J Pharmacol Toxicol Methods. 2007;56:131-144.

21. Nakashima H, Gerlach U, Schmidt D, et al. In vivo electrophysiological effects of a selective slow delayed-rectifier potassium channel blocker in anesthetized dogs: potential insights into class III actions. Cardiovasc Res. 2004;61:705-714.

22. Gallacher DJ, Van de Water A, van der Linde H, et al. In vivo mechanisms precipitating torsades de pointes in a canine model of drug-induced long-QT1 syndrome. Cardiovasc Res. 2007;76:247-256.

23. Thomas GP, Gerlach U, Antzelevitch C. HMR 1556, a potent and selective blocker of slowly activating delayed rectifier potassium current. $J$ Cardiovasc Pharmacol. 2003;41:140-147. 
24. Walker MJ, Curtis MJ, Hearse DJ, et al. The Lambeth conventions: guidelines for the study of arrhythmias in ischaemia infarction, and reperfusion. Cardiovasc Res. 1988;22:447-455.

25. Farkas A, Batey AJ, Coker SJ. How to measure electrocardiographic QT interval in the anaesthetized rabbit. J Pharmacol Toxicol Methods. 2004; 50:175-185.

26. Batey AJ, Coker SJ. Proarrhythmic potential of halofantrine, terfenadine and clofilium in a modified in vivo model of torsade de pointes. $\mathrm{Br} J$ Pharmacol. 2002;135:1003-1012.

27. Carlsson L, Abrahamsson C, Andersson B, et al. Proarrhythmic effects of the class III agent almokalant: importance of infusion rate, QT dispersion, and early afterdepolarisations. Cardiovasc Res. 1993;27:2186-2193.

28. Altman DG. Practical Statistics for Medical Research. 1st ed. London, United Kingdom: Chapman \& Hall; 1991.

29. Varró A, Baczkó I. Cardiac ventricular repolarization reserve: a principle for understanding drug-related proarrhythmic risk. $\mathrm{Br} J$ Pharmacol. 2011;164:14-36.
30. Berger RD, Kasper EK, Baughman KL, et al. Beat-to-beat QT interval variability: novel evidence for repolarization lability in ischemic and nonischemic dilated cardiomyopathy. Circulation. 1997;96: 1557-1565.

31. Hondeghem LM, Dujardin K, De Clerck F. Phase 2 prolongation, in the absence of instability and triangulation, antagonizes class III proarrhythmia. Cardiovasc Res. 2001;50:345-353.

32. Oros A, Beekman JD, Vos MA. The canine model with chronic, complete atrio-ventricular block. Pharmacol Ther. 2008;119:168-178.

33. Pedersen HS, Elming H, Seibaek M, et al. Risk factors and predictors of torsade de pointes ventricular tachycardia in patients with left ventricular systolic dysfunction receiving dofetilide. Am J Cardiol. 2007;100: 876-880.

34. Day CP, McComb JM, Campbell RW. QT dispersion in sinus beats and ventricular extrasystoles in normal hearts. Br Heart J. 1992;67:39-41.

35. Hondeghem LM. Relative contributions of TRIaD and QT to proarrhythmia. $J$ Cardiovasc Electrophysiol. 2007;18:655-657. 